\title{
Conceptual service architecture for adaptive mobile location services
}

\section{Schou, Saowanee}

\section{Published in:}

Proceedings of The International Conference on Information Networking, 2008

Link to article, DOI:

10.1109/ICOIN.2008.4472796

Publication date:

2008

Document Version

Publisher's PDF, also known as Version of record

Link back to DTU Orbit

\section{Citation (APA):}

Schou, S. (2008). Conceptual service architecture for adaptive mobile location services. In Proceedings of The International Conference on Information Networking, 2008 (pp. 118-122). IEEE.

https://doi.org/10.1109/ICOIN.2008.4472796

\section{General rights}

Copyright and moral rights for the publications made accessible in the public portal are retained by the authors and/or other copyright owners and it is a condition of accessing publications that users recognise and abide by the legal requirements associated with these rights.

- Users may download and print one copy of any publication from the public portal for the purpose of private study or research.

- You may not further distribute the material or use it for any profit-making activity or commercial gain

- You may freely distribute the URL identifying the publication in the public portal

If you believe that this document breaches copyright please contact us providing details, and we will remove access to the work immediately and investigate your claim 


\title{
Conceptual Service Architecture for Adaptive Mobile Location Services
}

\author{
Saowanee Schou \\ Center for Information and Communication Technologies \\ Technical University of Denmark \\ saowanee@cict.dtu.dk
}

\begin{abstract}
This paper presents a conceptual service architecture for adaptive mobile location services designed to be used on the next generation wireless network. The developed service architecture consists of a set of concepts, principles, rules and guidelines for constructing, deploying, and operating the mobile location services. The service architecture identifies the components required to build the mobile location services and describes how these components are combined and how they should interact. As a means of exploring the developed conceptual service architecture, an illustrative case study of a new-concept tracking service is chosen to demonstrate the applicability of the architecture. Through the case study, the service request and response processes will be illustrated. New possible service functions provided by the developed service architecture will be examined and discussed in comparison with the functions provided by the existing tracking services on the current network to show the usefulness and value of the developed service architecture.
\end{abstract}

\section{INTRODUCTION}

The existing mobile location services are typically available locally within the network of a specific operator or available on different networks of different operators in the same country. The way mobile location services are offered today is not compatible with the service environment of the next generation wireless network which will be open [1][2]. In the open service environment, the users will be able to access a mobile service regardless of geographical location, terminal model, access network, network operator and service provider $[1][2][3][4]$. Different services will be provided by different service providers on a common IPv6 network, and these services will be available to all users on the network, regardless of which part of the network they connect from [3][4]. Location information, user profile, and billing and charging information can be transferred between administrative domains, operators and service providers [1][3]. Both seamless roaming and universal access is expected to be achieved in the next generation wireless network [2][5].

Providing mobile location services on the next generation wireless network requires a new service architecture that fits in an open service environment and which allows the coexistence of a number of stakeholders performing various roles. The new service architecture must support universal service access and allow the end-users to access services independently of the physical location, type of access network and terminal model being used by the user.
This paper presents a conceptual service architecture for adaptive mobile location services which is designed to be used in the open service environment of the next generation wireless network. The architecture supports a provision of new-concept services which have not been possible to provide using the existing service architecture on the current network, e.g. the services that allow users to access a service based on the location information of other users on the IPv6 network. For example, the location-based information service may be provided based on the location of other users at the globallevel instead of based on the current location of the user or the location manually defined by the user like the services available today. The mobile location services that are currently only available in the local domain (e.g. tracking services) will possibly be made available globally (e.g. tracking the location of a child who is traveling abroad).

The "help you out" scenario presented in the following represents example usage cases of new-concept mobile location services based on the developed service architecture presented in this paper. The types of services are indicated in the footnotes.

Scenario: Help you out "Sara is 12 years old and she is traveling alone to Tokyo for studying Japanese. Sara has not been in Tokyo before but her father was there last year. He wants to help Sara with practical things such as where to go and what to buy and which areas are safe and which areas should be avoided. Sara appreciates her father's help and she shares her location information with her father and remains open for his suggestions.

When Sara arrives at the Narita airport, her father gets an alert message that she has arrived safely ${ }^{1}$ and now she needs to go through all the check out processes and then get her luggage. While Sara is managing the practical things at the airport, her father helps her finding a taxi by locating the location of the taxi in the vicinity of the airport and reserves one for Sara ${ }^{2}$. The service sends reservation information to Sara including the taxi number and real-time navigation map to the taxi's location ${ }^{3}$. Sara gets her luggage and the taxi is ready for her. Sara is getting into a taxi and her father is monitoring her location all the way to the hotel $^{4}$. Sara is jet-lagged and she wants to rest. She asks her father to make a traveling plan to the school for her tomorrow. Sara's father accesses a navigation service and makes prenavigation for her. He chooses that the navigation service should

\footnotetext{
${ }^{1}$ Pre-tracking service.

${ }^{2}$ Location-based information service.

${ }^{3}$ Navigation service.

${ }^{4}$ Tracking service.
} 
be alerted at Sara's terminal at 8 O'clock tomorrow morning 5 . Sara's father can access any information related to the location of Sara. Sara shares her location with her father and he can help her check all the conditions based on Sara's location (e.g. weather, traffic, safety/danger zones, etc). Sara's father is not concerned about Sara, as he can see that she is safe and managing her life fine in Tokyo".

The components required to build the conceptual service architecture for providing new-concept mobile location services are introduced in section II together with descriptions of how these components are combined and how they should interact. An illustrative case study of a new-concept tracking service is made in section III as a means of exploring and demonstrating the applicability of the developed conceptual service architecture. Section IV discusses the usefulness of the new-concept tracking service based on the developed service architecture in comparison with the existing tracking services on the current network. Concluding remarks are given in section $\mathrm{V}$.

\section{CONCEPTUAL SERVICE ARCHITECTURE FOR ADAPTIVE MOBILE LOCATION SERVICES}

This section presents the conceptual service architecture for adaptive mobile location services which is designed to be used in the open service environment of the next generation wireless network. One of the important features of the designed architecture is adaptability. Adaptability is envisioned by WWRF as one of the keys to the success of any service beyond year 2010 [6] and one of the service capabilities that should be made available on the next generation wireless network suggested by ITU [1]. Adding adaptability to the future mobile location services will therefore be one way of increasing the possibility of the service being a success.

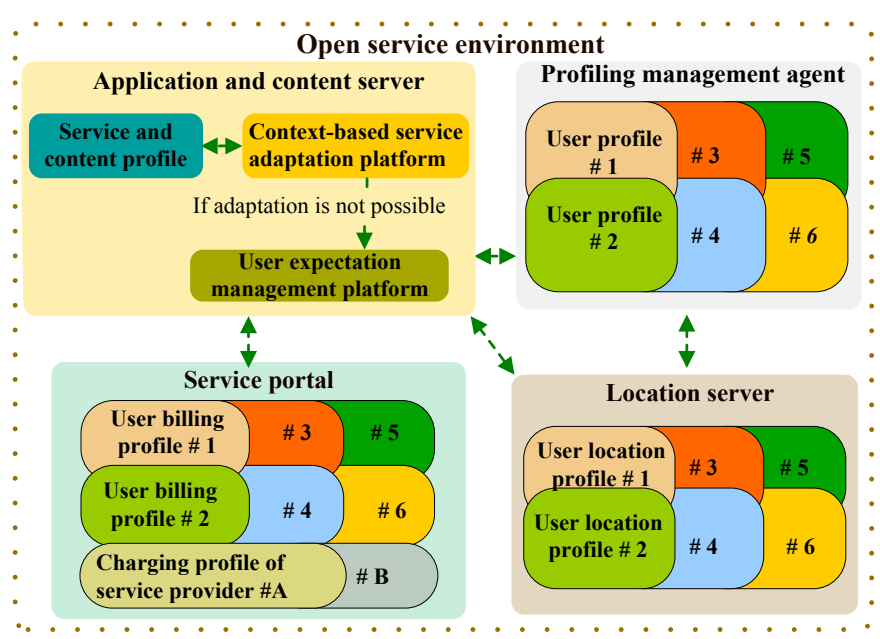

Figure 1: New conceptual service architecture for adaptive mobile location services on the next generation wireless network. The conceptual IPv6-based location method proposed in [9] may be used for determining the location of all users on the unified IPv6 network, and the location information is maintained by the location server. For simplicity, the infrastructure needed for location determination is not shown in the figure.

\footnotetext{
${ }^{5}$ Pre-navigation service.
}

Figure 1 illustrates the components that form the service architecture, and the descriptions and roles of the individual components are given in the following.

The service portal handles session management, requests handling, authentication of subscribers, and manages the billing and charging. The service portal contains the "user billing profile" and "service provider charging profile". When the user accesses the service, the service usage will be recorded and the billing and charging reports will be updated in the user billing profile and service provider charging profile, respectively. The user and service provider can access and check their profiles but editing and deleting of the profiles is not allowed. The user billing profile contains the actual information of the user, which is required for billing management such as the real name, real address, telephone number, credit card number and a list of subscribed services. The service portal allows users (or subscribers) of different network operators to access the services of different service providers from anywhere on the all-IPv6 network with the feasibility of managing the billing for the users and revenue sharing between different stakeholders. The service portal is placed in every domain and it is owned and managed by the network operator who administrates the domain. The service portal makes it easy for the user and service providers, as the user can use different services without having to pay different bills for different service providers and the service providers do not have to handle the billing management of different users but instead let the network operator handle this task.

The application and content server handles different tasks from providing the service and content to the user and adapting the service behaviors and content to best fit with the user's requirements/preferences in a specific context of use, to managing the user expectations towards the service. This server is owned and managed by the service provider. The context-based service adaptation platform plays an important role in managing the user experience in the usage stage of the service, as the context plays an important role in defining how the user will experience the service [7]. The service adaptation is made based on the context-dependent user's requirements and preferences and the conditions defined in the user profile ${ }^{6}$ and the service and content profile. The service and content profile is created by the service provider and maintained in the application and content server, which is owned by the service provider. This service and content profile contains information about what the service provides and what can be adapted and cannot be adapted in different contexts and situations.

Another task of the application and content server is to manage the user expectation, and this task is handled by the user expectation management platform. The main task of this platform is to inform the user about the service (e.g. if the service is temporarily unavailable, new features, new service update, etc.) and to inform the user of the reason and further

\footnotetext{
${ }^{6}$ The user profile is merely a conceptual entity representing a unique lifestyle and current context surrounding and situation of a user. The user profile contains details of the user and his personal requirements in a form that can be used by the system to deliver the service that match the user's requirements in particular contexts of use and situations [12].
} 
suggestions in the case that the service adaptation requested by the user cannot be made (e.g. 3D navigation is not possible because the user's terminal does not support 3D display). This is the platform that controls the user experience by providing an understanding of what the user can and cannot expect from the service. This approach prevents the user from generating unrealistic expectations that the service cannot live up to.

The profiling management agent maintains, manages and updates the user profiles of registered users and also handles authentication and authorization. The profiling management agent has been added to facilitate the concept of open service environment of the next generation wireless network where the information resources (e.g. user profile and location information) should be reusable and sharable across networks [1]. As different services may require similar or identical information related to the users and their current contexts, the profiling management agent provides a great benefit to the users by allowing them to share and reuse their profiles or parts of the profiles with different service providers without having to create and update the same information many times than necessary. The service providers also benefit from the profiling management agent, as they do not have to implement a platform for handling the user profiles of different users, instead letting the profiling management agent handle this task. The profiling management agent can be placed anywhere on the all-IPv6 network and it is owned by a new stakeholder, which in this paper is called the "profiling broker". This agent acts as a broker handling the usage and sharing of the information in the user profile according to privacy rules defined by the users. Based on the open service environment concept of the next generation wireless network, the users should be able to access, edit or delete their user profiles anywhere and any time they desire [1].

The location server maintains the location information of all the users in the domain. This server is owned and managed by the domain owner (network operator). There should be at least one location server in every domain. In the location server, the location of individual users is stored in a profile called the user location profile. This profile maintains the actual location of the user and the privacy rules of using and sharing this information is defined by the users. As the mobile user may travel into different domains administrated by different management entities or different network operators, it should be possible to exchange the user location profile between different network operators according to the user mobility. For example, if the user moves from domain A to domain $\mathrm{B}$, the transfer of user location profile from domain $\mathrm{A}$ to $\mathrm{B}$ should be possible. This requires new mechanisms for handling the user location profile transfer process and the agreements between network operators.

The ways the components in the service architecture, presented in figure 1 , interact with each other vary depending on the types of service, adaptation conditions, privacy rules, and context. However, the typical service requests and responses are as follow. The user accesses the service via the service portal. The service portal authenticates the service request, records the service usage and updates the user billing profile and provider charging profile. The service request is forwarded to the application and content server. The application and content server asks for the user profile from the profiling management agent. The location server may send the most up to date location information of the user to the profiling management agent depending upon the rules of using and sharing of location information defined in the user location profile. In the case that the service (e.g. navigation service) requires real-time location information, the application and content server may request the real-time location information directly from the location server. The application and content server then delivers the requested service back to the user.

\section{NEW-CONCEPT TRACKING SERVICES}

The tracking services based on the existing service architecture on the current mobile networks are typically available locally within the same kind of mobile network technology in the same domain or available for the subscribers of different operators in the same country. The concept of tracking services on the developed conceptual service architecture presented in this paper is compatible with the service environment of the next generation wireless network, where the service is available in the global-level and supports universal access, meaning that the user can access the same tracking service regardless of geographical location, terminal model, access technology, network operator and service provider. Figure 2 demonstrates the request/response sequences of the tracking service based on the "help you out" scenario presented previously in the introduction section.

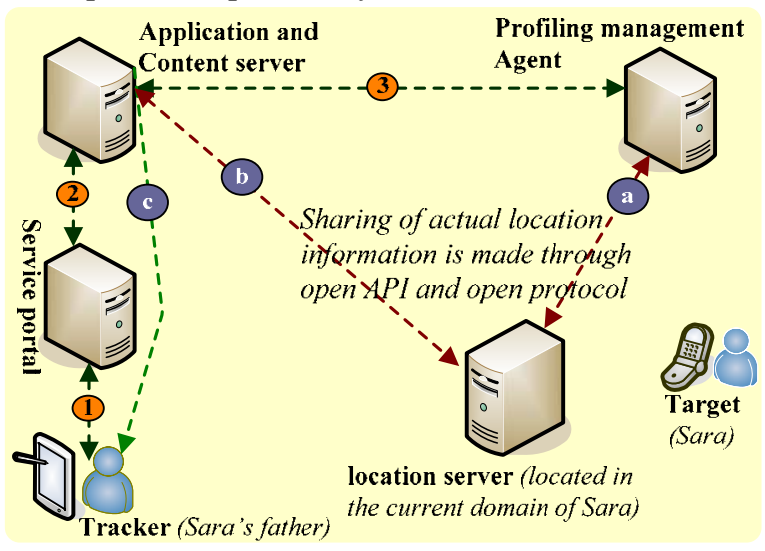

Figure 2: Interactions between different components in the conceptual service architecture in the case of a global-level tracking service. The user profiles of the tracker and the target are assumed to be maintained in the same profiling management agent. The tracker and the target are in different domains and different countries. Each domain has a location server which maintains the actual location of all users on the domain and the actual location of the tracker and the target in the scenario are maintained by different location servers.

Based on the "help you out" scenario, the tracking service is triggered by the current use context of the target (i.e. Sara's current location). The service is activated when Sara arrives at Narita airport. In this case, Sara's father has defined beforehand that the service must send notification of arrival to him when Sara arrives in Japan. The tracking service is continuously used by Sara's father until she arrives at the hotel. In this case, Sara's father is the tracker and Sara is the 
tracking target. The processes of service activation, requests and responses of the tracking service presented in the scenario are as follow.

1. The tracker sends a service request to the application and content server via the service portal (Route 1). The service portal authenticates the request and records the service usage for billing and charging management.

2. The service portal forwards the service request to the application and content server (route 2). Based on the scenario, the tracker defines that he wants the service to be activated when the target enters a pre-defined context (i.e. the target has arrived in the pre-defined location (Narita Airport)).

3. The application and content server asks the profiling management agent for the user profiles of the tracker and the target (assuming that the user profile of the tracker and the target are maintained in the same profiling management agent). The profiling management agent processes the privacy control and delivers the information required for the requested service to the application and content server (via route 3 ). The profile of the tracker will be used for adapting the service (e.g. service visualization, user interface, etc) to the tracker's requirements/preferences in his current use context, and the location information of the target in her user profile will be used for service triggering (i.e. service behavior adaptation).

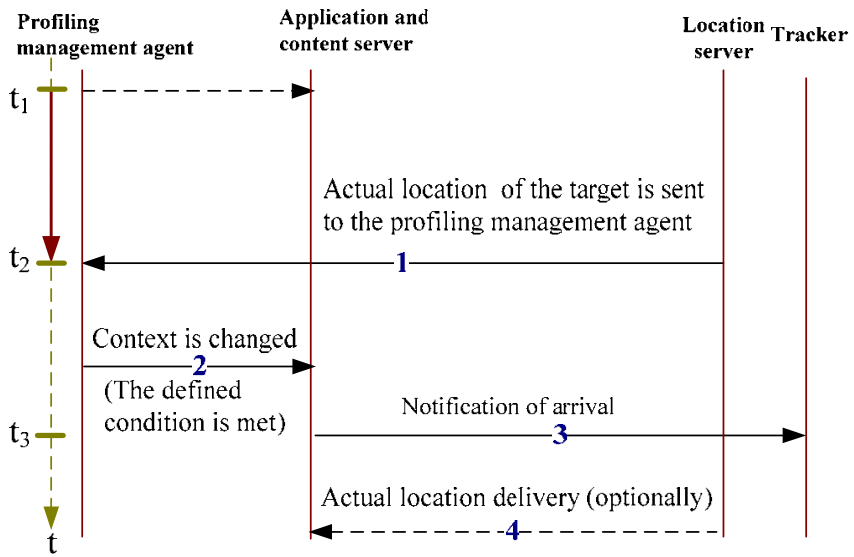

Figure 3: Interactions between the components in the service architecture from the start of the monitoring period (t1) to the point where the arrival alert is sent out to the tracker $(\mathrm{t} 3)$. The period from $\mathrm{t} 1$ to $\mathrm{t} 2$ is the monitoring period. The target enters the pre-defined area at $\mathrm{t} 2$ and notification of arrival is sent to the tracker at $\mathrm{t} 3$.

After the tracker has requested the tracking service, the profiles of the tracker and the target are sent to the application and content server. Based on the scenario, the tracker defines the service behavior by setting that the tracking function should be triggered when the target has entered the predefined area. The period from this point to the point when the target is in the pre-defined area is, in this paper, referred to as the "monitoring period" (i.e. the period where the service is waiting for activation depending on the conditions defined by the tracker). The service will remain in this period as long as the target is not in the pre-defined area. Figure 3 illustrates the interactions between the components in the service architecture from the start of the monitoring period to the point where the tracking function is activated and the target is tracked continuously by the tracker. The tracking function will be activated when the target is in the pre-defined area (i.e. arrived at Narita Airport). The service activation processes are as follows.

1. When the target is at the Narita airport and turns on her mobile terminal $\left(t_{2}\right)$, one of the available indoor location methods (e.g. the method presented in [8]) determines her location and sends the location information to the location server. The location server then sends information update to the profiling management agent where the target has registered (route a in figure 2 and process \# 1 in figure 3). The sharing of location information between the location server and application and content server is made through open API and open protocol ${ }^{7}$.

2. The profiling management agent detects the change of current use context of the target (the target enters a new location). The new location information of the target is then sent to the context-based service adaptation platform located in the application and content server (process \# 2 in figure 3). This information will be used for service triggering.

3. The condition set by the tracker (i.e. the target is entering the pre-defined area) is met, and the tracking function is activated. At this point $\left(\mathrm{t}_{3}\right)$, the notification of arrival is sent to the tracker and the global-level tracking service begins (process \# 3 in figure 3 ).

4. Based on the "help you out" scenario, from the point where the notification of arrival is sent, the target will be continuously tracked by the tracker (via route $\mathrm{c}$ in figure 2). As real-time location information is needed from this point, the location information of the target may optionally be sent directly from the location server to the application and content server (route $b$ in figure 2 and process \# 4 in figure 3).

Based on the scenario, the location of Sara will be tracked from the point where she arrives at the airport, during the check-out and luggage collection, and while taking a taxi to the hotel. The tracking service will be withdrawn when Sara arrives at the hotel.

\section{USEFULNESS OF THE DESIGNED SERVICE ARCHITECTURE}

Based on the demonstration of the tracking service presented above, the conceptual service architecture can in principle provide several new functions for the new-concept tracking service compared to the functions for the existing tracking services. Global-level is one of the main advantages of the conceptual service architecture, and global-level tracking is one of the new possible functions of the developed conceptual service architecture. This function is not possible with the current service architecture in the current service environment and on the current network, due to the lack of open standards and open service environment, and the fact that the mechanism for sharing the required information (e.g. location and user profile) is not available.

The lack of open standards and open service environment is one of the shortcomings of the existing mobile location

\footnotetext{
${ }^{7}$ An open protocol is an application-level protocol for querying the location of mobile terminals independent of underlying network technology. The open protocol serves as the interface between a location server and profiling management agent or application and content server [13].
} 
services. The availability of the existing mobile location services is depending on the geographical location, service provider, network operator, and terminal. For some tracking services the users are required to have a specific terminal in order to be able to access the service, and this service is only available locally within a specific geographical area and a specific network, and the tracking is only possible if the tracker and the target are subscribers of the same network operator and service provider.

Adaptability is another useful capability of the conceptual service architecture. Based on the "help you out" scenario, the context-based service adaptation will adapt the service behavior based on the task defined by the tracker. In this case, the tracker has defined that he wants to be notified when the target is in the pre-defined area. The service will be activated and the notification will be sent to the tracker when the conditions set by the tracker are met. With this service function, the tracker does not have to manually track the location of the target, but rather let the tracking service monitor the location of the target and notify the tracker when condition set by the tracker is met. This function is currently available in some of the tracking services offered in Japan and South Korea but not for the services in Western Europe.

Another advantage of the tracking service based on the conceptual service architecture is that it is available in indoor environments (e.g. airports). This can be realized by the use of Bluetooth location infrastructure [8] and the conceptual IPv6based location method proposed in [9]. The lack of capability of providing high accurate location in indoor environments and dense urban areas is one of the main problems limiting the success of the tracking services offered today [10]. With the conceptual IPv6-based location solution, and indoor location infrastructure applying short- and medium-range wireless networks [8][9], the lack of high accuracy location information in closed environments can be compensated.

Norman has stated in the book "Emotional design" [11] that the service that almost always guarantees success is the service that provides social interaction and emotional connection between people. The global-level tracking service presented in the "help you out" scenario provides social interactions and emotional connections between people (father and his child). Naturally, the father is concerned about his child, especially when she is a girl and alone in a foreign country. The child is likely to be nervous when she is alone in an unfamiliar area, and she wants to feel safe. The global-level tracking service allows the father to see what is going on with his child and at the same time he can help his child handle some of her tasks remotely as presented in the scenario. This service has a high potential of being a success if it is used between trust parties. However, it involves a higher level of complexity to realize this service concept compared to the tracking service that is available locally. In order to realize the services presented in the scenario, exchanging of user profile, location information, and billing and changing information between administrative domains is required, and new business agreements and new business models are needed to be developed.

\section{CONCLUSION}

This paper presents a conceptual service architecture for adaptive mobile location services to be used in the open service environment of the next generation wireless network. In the designed service architecture, the service portal and profiling management agents play important roles in realizing the concept of one network many services. The context-based service adaptation platform, user profile and service and content profile play important roles in adapting the service to best fit with the user requirements in a particular context of use. In the designed architecture, the user profile is handled by the profiling management agent and not by the service provider like the case today. The service provider can only utilize the user profile when the user wants to use the service. This will minimize the privacy concern towards the use of any mobile service. It is obvious that the profiling management agent plays an important role in providing mobile services in the future. The profiling management agent must be trustworthy and the users should feel safe and comfortable allowing the agent to protect the use of their personal information as well as their location information. The developed service architecture supports the deployment of new kinds of mobile location services that have not been possible on the current network. The services such as globallevel tracking service and location-based information service that allows the user to access the information utilizing the location information of other users on the all-IPv6 network will be possible.

\section{REFERENCES}

[1] ITU-T (2005), ITU-T NGN FG Proceedings Part II, Next Generation Network Global Standards Initiative, ITU.

[2] WWRF (2006), "Visions of system concepts for wireless world", Wireless World Research Forum (WWRF), Issue 2.1.

[3] Reynolds T. \& Jin-Kyu J. (2004), "Shaping the future mobile information society: The case of the republic of Korea", Case study report: SMIS/07Strategy and Policy Unit, International Telecommunication Union (ITU), February, pp 24.

[4] Chen et al. (2007), "Reconfigurable Architecture and Mobility Management for Next-Generation Wireless IP Networks", IEEE transaction on wireless communication, Vol. 6. Issue 8, pp. 3102-3113.

[5] Chen Y. and Yang Y. (2007), "A new 4G architecture providing multimode terminals always best connected services", IEEE Wireless Communications, Vol.14 Issue.2, pp.36-41.

[6] Uusitalo M. (2006), "Global visions for the future wireless world from the WWRF", IEEE Vehicular Technology magazine, Vol.1 Issue.2, pp. 4-8.

[7] Hiltunen M., Luakka M. and Luomala J. (2002), "Mobile user experience", IT press, Finland.

[8] Thongthammachart S. and Olesen H. (2003) Bluetooth enables in-door mobile location services, 57th IEEE Semiannual Vehicular Technology Conf. (VTC'2003 spring), Jeju, Korea, April, session 8H, paper no. 3.

[9] Schou S. T. and Olesen H. (2005), "Detection of mobile user location on next generation wireless networks", Journal of Internet Technology Special Issue on "Heterogeneous IP Networks", vol: 6, pp. 273-279.

[10] De La Vergne et al. (2006), " Forecast: GPS in Mobile Devices Worldwide 2004-2010", Market analysis report, ID Number: G00144746, Gartner, Inc.

[11] Norman D. A. (2004), "Emotional Design: Why We Love (Or Hate) Everyday Things", New York: Basic books, p.19.

[12] ETSI (2005), "Human Factors (HF): User Profile Management", EG 202 325 V1.1.1, European Telecommunications Standards Institute.

[13] OMA (2004), "Mobile Location Protocol (MLP)", Candidate Version 3.1, Work in progress document, Open Mobile Alliance. 